\title{
miR-21 promotes migration and invasion by the miR-21-PDCD4-AP-1 feedback loop in human hepatocellular carcinoma
}

\author{
QIN ZHU, ZHIMING WANG, YU HU, JINDONG LI, XINYING LI, LEDU ZHOU and YUN HUANG
}

Department of General Surgery, Xiangya Hospital, Central South University, Changsha, Hunan 410008, P.R. China

Received December 14, 2011; Accepted January 16, 2012

DOI: $10.3892 / 0 r .2012 .1682$

\begin{abstract}
Distant migration and invasion is the main contributor to the death of cancer patients and miRNAs have been implicated in these processes. In the present study, we identified the role of microRNA-21 (miR-21) in hepatocellular carcinoma (HCC) migration and invasion and determined its underlying regulatory mechanism. miR-21 was significantly upregulated in HCC tissues and cell lines, compared with adjacent non-tumor tissues and normal hepatic cells. miR-21 upregulation was associated with the capacity of tumor migration and invasion in HCC. The expression level of miR-21 was inversely correlated with the protein expression level of a previously identified target gene, programmed cell death 4 (PDCD4). In HepG2 cells, inhibition of miR-21 expression repressed cell migration and invasion by upregulating both mRNA and protein levels of PDCD4 and downregulating key downstream signaling pathway molecules, including phosphoc-Jun, matrix metalloproteinases (MMP)-2 and MMP-9. Moreover, activation protein 1 (AP-1) could directly activate miR-21 transcription. Taken together, these results provide evidence that miR-21 promotes migration and invasion in HCC through the miR-21-PDCD4-AP-1 feedback loop, suggesting that targeting the miR-21-PDCD4-AP-1 loop may represent a promising strategy in the management of HCC.
\end{abstract}

\section{Introduction}

Hepatocellular carcinoma (HCC) is one of the most common malignant tumors diagnosed in Asian countries, and is the second leading cause of cancer mortality in China. Surgical resection remains the main radical treatment of choice for

Correspondence to: Dr Zhiming Wang, Department of General Surgery, Xiangya Hospital, Central South University, Changsha, Hunan 410008, P.R. China

E-mail: zmwangxy@126.com

Key words: microRNA-21, programmed cell death 4, activation protein 1 , hepatocellular carcinoma, invasion, migration
HCC. HCC has poor prognosis and the 5-year survival rate after operation has been reported to range from only $36-50 \%$ (1). Like many other cancers, distant metastasis is the main contributor to poor prognosis. Migration and invasion of HCC is a multi-phase developmental process that involves changes in multi-gene signatures. Therefore, early prevention of HCC invasion and metastasis has become the primary focus of many research studies. Some molecules have been found to play an important role in HCC invasion and metastasis, such as p53 mutation (2), MDM-2 (3), telomerase (4), $\beta$-catenin (5) and VEGF (6). These molecules represent promising therapeutic targets for preventing HCC metastasis; however, their specificity and sensitivity need to be further studied.

MicroRNAs (miRNAs) are a class of small non-coding RNAs that are 18-25 nucleotides in length and negatively regulate gene expression by binding to the 3 -untranslated region (3'-UTR) of specific target messenger mRNAs (mRNAs), which in turn causes mRNA degradation or translational repression (7). Aberrant miRNA expression has been linked to human diseases, including cancer. As an oncogene, microRNA-21 (miR-21) was found to be overexpressed in a variety of malignancies, including cholangiocarcinoma (8), breast cancer (9), lung cancer (10), prostatic carcinoma (11) and colon carcinoma (12). miR-21 has also been implicated in multiple malignancyrelated processes, including cell proliferation, apoptosis, invasion and metastasis, by downregulating the expression of specific target genes, such as phosphatase and tensin homologue (PTEN), tropomyosin 1 (TPM1), programmed cell death 4 (PDCD4) and B-cell lymphoma 2 (Bcl-2) (13-16). Although the miR-21-PDCD4 axis has been addressed in other cancer types $(19,24)$, the involvement of miR-21 in the migration/invasion of HCC and its underlying mechanism is not yet known.

As a previously identified target gene of miR-21, PDCD4 harbors a highly conserved binding site for miR-21 in its 3'-UTR by using bioinformatics tools. PDCD4 is a tumor suppressor that plays an important role in regulating apoptosis, invasion and metastasis $(17,18)$. In pancreatic ductal adenocarcinoma, inhibition of miR-21 reduces proliferation and increases cell death by increasing PDCD4 (19). We hypothesized that miR-21 promoted migration and invasion of HCC by a mechanism involving PDCD4. This is the first report to demonstrate that miR-21 indirectly activates activation protein 1 (AP-1)dependent transcription by repressing PDCD4, and stimulates 
migration and invasion in HCC. Meanwhile miR-21 transcription is directly triggered by AP-1. Together, these data suggest that the miR-21-PDCD4-AP-1 feedback loop sustains the biological effects of miR-21 in HCCs.

\section{Materials and methods}

Patients and tissue samples. Paired HCC tumor tissues and adjacent non-tumor liver tissues were collected from $16 \mathrm{HCC}$ patients who underwent hepatectomy between October 2010 and March 2011 at Xiangya Hospital (Changsha, China), after obtaining informed consent and verification by an experienced pathologist. Tissue samples were frozen in liquid nitrogen immediately after resection and stored at $-80^{\circ} \mathrm{C}$ until use. No patient in the current study received chemotherapy or radiation therapy before the surgery. The pathological differentiation was defined according to the Edmondson system. The clinical stage was defined according to the tumor-node-metastasis (TNM) staging system (6th UICC) (20). Among the patients, eight had primary HCC lesions accompanied by intrahepatic metastases, vascular invasion, or capsular invasion, and eight had no metastasis/invasion found at surgery. This study was approved by the Human Research Ethics Committee of Xiangya Hospital. Clinicopathologic characteristics of these patients, including gender, age, differentiation, metastasis/ invasion, TNM staging, hepatitis-B surface antigen ( $\mathrm{HBsAg}$ ), cirrhosis, and $\alpha$-fetoprotein (AFP), were collected and listed in Table I.

Cell culture and transfection. Human normal liver cells L02 and human hepatocellular carcinoma cell lines HepG2, MHCC97H, Bel7402 and Huh7 were obtained from the Cell Center at the Xiangya School of Medicine. All cell lines were grown in DMEM medium supplemented with $10 \%$ fetal bovine serum (FBS), $100 \mathrm{IU} / \mathrm{ml}$ penicillin and $100 \mu \mathrm{g} / \mathrm{ml}$ streptomycin in humidified incubator at $37^{\circ} \mathrm{C}$ with $5 \% \mathrm{CO}_{2}$.

The miR-21 inhibitor and PDCD4 siRNA were purchased from GenePharma (Shanghai, China). HepG2 cells were plated in 6-well plates at $50 \%$ confluence the day before transfection. The next day, $50 \mathrm{nM}$ of siRNA or miR-21 inhibitor was transfected into HepG2 cells using Lipofectamine 2000 (Invitrogen, Carlsbad, CA, USA) according to the manufacturer's instructions. Total-RNAs or proteins were prepared at 48 or $72 \mathrm{~h}$ post-transfection and used for quantitative reverse transcription (qRT)-PCR or western blot analysis, respectively. For the PDCD4 siRNA and miR-21 inhibitor combination experiments, HepG2 cells were first transfected with miR-21 inhibitor $(50 \mathrm{nM})$ or inhibitor negative control (NC) $(50 \mathrm{nM})$. At $48 \mathrm{~h}$ later, these cells were co-transfected with PDCD4 siRNA (50 nM) and miR-21 inhibitor (50 nM) or control oligos for another $72 \mathrm{~h}$. Cells were used for transwell assays or qRT-PCR/western blot analyses.

qRT-PCR analysis of miR-21 and PDCD4, matrix metalloproteinases (MMP)-2, and MMP-9 mRNA expression. Total-RNA was extracted using TRIzol reagent (Invitrogen) according to the manufacturer's protocol. Expression of miR-21 was determined by the NCode EXPRESS SYBR-GreenER miRNA qRT-PCR kit (Invitrogen), and normalized to U6 snRNA using the $2^{-\Delta \Delta C t}$ method (21). PDCD4, MMP-2 and MMP-9 mRNA
Table I. Clinicopathologic characteristics of the HCC patients.

Characteristics

$\begin{array}{lc}\text { Age, years (mean } \pm \mathrm{SD} \text { ) } & 49.9 \pm 10.5 \\ \text { Gender (male/female) } & 13 / 3 \\ \text { Differentiation (well/moderate/poor) } & 2 / 11 / 3 \\ \text { Metastasis (positive/negative) } & 8 / 8 \\ \text { TNM stage (I-II/III-IV) } & 7 / 9 \\ \text { HBsAg (positive/negative) } & 14 / 2 \\ \text { Cirrhosis (positive/negative) } & 13 / 3 \\ \text { AFP (positive/negative) } & 10 / 6\end{array}$

TNM, tumor-node metastasis system; HBsAg, hepatitis-B surface antigen; AFP, $\alpha$-fetoprotein.

expression was quantified using the SYBR PrimeScript RT-PCR kit II (Takara Bio, Inc., Shiga, Japan) and normalized to GAPDH using the $2^{-\Delta \Delta \mathrm{Ct}}$ method. The PCR primers were as follows: PDCD4, forward, 5'-TGGATGTCCCACATTCATACTCTG-3' and reverse, 5'-TCTGGTTTAAGACGACCTCCATCT-3'; MMP-2, forward, 5'-TGGCGATGGATACCCCTTT-3' and reverse, 5'-TTCTCCCAAGGTCCATAGCTCAT-3'; MMP-9, forward, 5'-TGCCCGGACCAAGGATACAG-3' and reverse, 5'-TCAGGGCGAGGACCATAGAG-3'; c-Jun, forward, 5'-ATC CTGAAACAGAGCATGAC-3' and reverse, 5'-TTGCTGG ACTGGATTATCA-3'; GAPDH, forward, 5'-TCAACGAC CACTTTGTCAAGCTCA-3' and reverse, 5'-GCTGGTGGT CCAGGGGTCTTACT-3'; miR-21, forward, 5'-TAGCTTAT CAGACTGATG-3' and reverse, 5'-TGGTGTCGTGGAG TCG-3'; U6, forward, 5'-CTCGCTTCGGCAGCACA-3' and reverse, 5'-AACGCTTCACGAATTTGCGT-3'. All qRT-PCR reactions were performed in triplicate.

Western blot analysis. For tissue sample, $100 \mathrm{mg}$ of liquid nitrogen-frozen tissue was ground into powder with a precooled mortar and pestle. Protein extracts of cells or frozen tissue powder were prepared by incubating in a RIPAlysis buffer (50 mM Tris- $\mathrm{HCl}$, pH 7.4; 150 mM NaCl; $1 \%$ Triton $\mathrm{X}-100$; $1 \%$ sodium deoxycholate; $0.1 \%$ SDS; 1 nM EDTA; $1 \%$ leupeptin) (Beyotime). Protein concentration was determined by the BCA protein assay kit (Thermo Scientific, Waltham, MA, USA). A total of $30 \mu \mathrm{g}$ of proteins were separated on $10 \%$ SDS-PAGE, and transferred to PVDF membranes (Millipore, Billerica, MA, USA). After blocking with 5\% non-fat milk at $37^{\circ} \mathrm{C}$ for $1 \mathrm{~h}$, membranes were incubated with the appropriate primary antibodies, including anti-PDCD4 (Abcam, Cambridge, UK), anti-c-Jun (Cell Signaling Technology, Irvine, CA, USA), anti-phospho-c-Jun (Cell Signaling Technology) or anti-GAPDH (G9545; Sigma-Aldrich, St. Louis, MO, USA). For reblotting, PVDF membranes were first stripped in a buffer containing glacial acetic acid at $37^{\circ} \mathrm{C}$ for $60 \mathrm{~min}$, then blocked and probed with a second primary antibody. After washing with Tris buffer, PVDF membranes were incubated with horseradish peroxidase-labeled goat antirabbit $\mathrm{IgG}$ at $37^{\circ} \mathrm{C}$ for $1 \mathrm{~h}$. Signals were visualized by using enhance chemiluminescence (GE Healthcare, Waukesha, WI, USA) and X-ray films. 
A
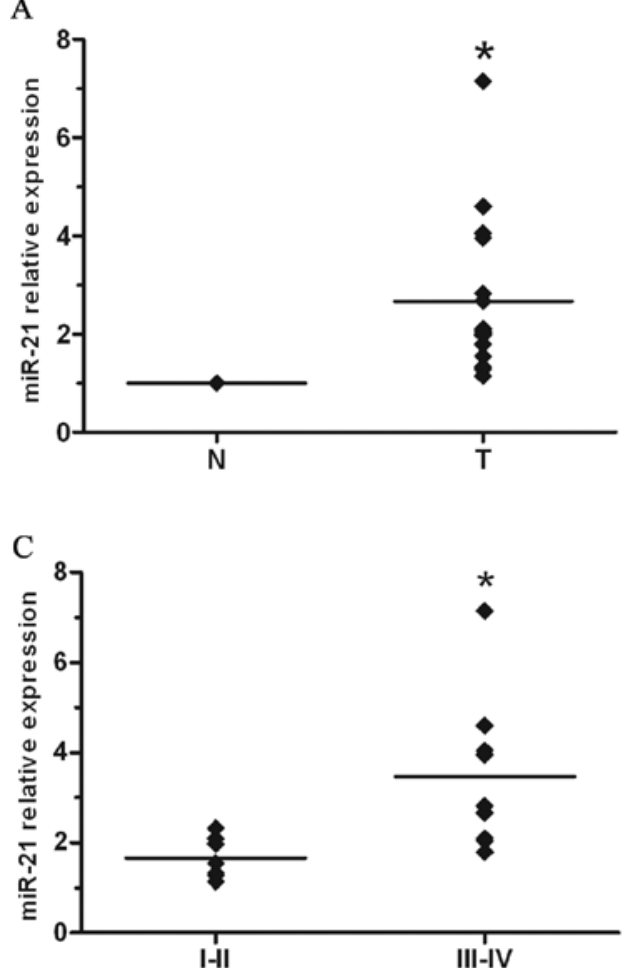

$\mathrm{B}$

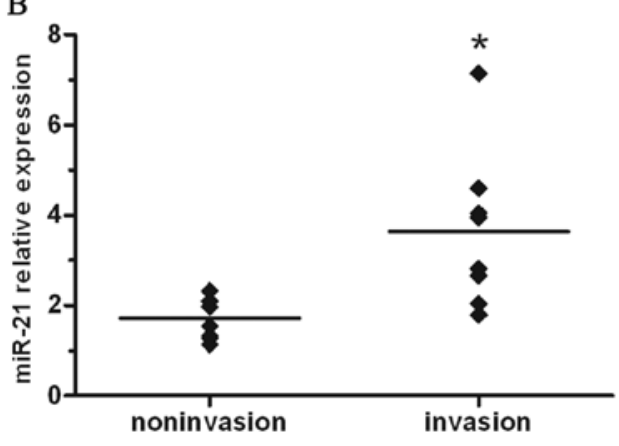

$\mathrm{D}$

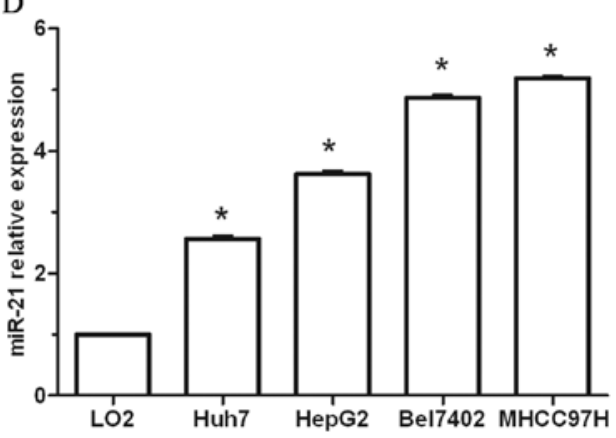

Figure 1. Overexpression of miR-21 was significantly upregulated in HCC tumor tissues/cell lines and was associated with increasing invasive capacity in HCC tissues/cell lines. (A) Relative miR-21 expression levels in tumor tissues and adjacent non-tumor tissues. miR-21 expression was determined in 16 paired HCC tissues by qRT-PCR. The expression levels of miR-21 relative to U6 snRNA were normalized to those detected in the adjacent non-tumor tissue of each matched pair. N, non-tumor tissue; T, tumor tissue. (B) Relative miR-21 expression levels in non-invasive and invasive HCC tumor tissues. (C) Relative miR-21 expression levels in patients with stage III-IV and patients with stage I-II. (D) Relative miR-21 expression levels in HCC cell lines with different invasive capacity (MHCC97H>Bel7402>HepG2>Huh7) and in normal hepatic cells (L02). ${ }^{*} \mathrm{P}<0.05$.

Cell migration and invasion assay. Migration of HepG2 cells was evaluated using transwell chambers with $8 \mu \mathrm{m}$ pore filters (Corning, Inc., Corning, NY, USA). At 48 or $72 \mathrm{~h}$ post-transfection, the miR-21 inhibitor and/or PDCD4 siRNA-transfected cells were trypsinized and $5 \times 10^{4}$ cells were plated on the upper chamber in serum-free medium. DMEM medium containing $10 \%$ FBS was added as a chemoattractant to the lower chamber. After incubating for $12 \mathrm{~h}$ at $37^{\circ} \mathrm{C}$ nonmigrating cells were removed with cotton swabs. Cells that migrated to the bottom of the membrane were then fixed with $95 \%$ ethanol, stained with $1 \%$ eosin for $10 \mathrm{~min}$ at $37^{\circ} \mathrm{C}$, and washed twice with PBS. Stained cells were visualized under a microscope (high-power field), and the number of cells counted in five random fields were averaged. For the HepG2 invasion assay, $5 \times 10^{4}$ post-transfected cells were added to the upper chamber that had been pre-coated with $30 \mu \mathrm{g}$ Matrigel (BD Biosciences, Franklin Lakes, NJ, USA). Then, DMEM medium containing $10 \% \mathrm{FBS}$ was added to the lower chamber. Cells were incubated for $24 \mathrm{~h}$ at $37^{\circ} \mathrm{C}$, and then non-invading cells were removed with cotton swabs. Invaded cells were fixed, stained and counted.

Statistical analysis. Each experiment was performed at least three times. Statistical analysis was performed using the SPSS software version 13.0 (SPSS Inc., Chicago, IL, USA). Values were expressed as mean \pm standard error of the mean (SEM). Differences/correlations between the two groups were calculated with the Mann-Whitney U test, Student's t-test, or
Pearson's test. Multiple group comparisons were analyzed using ANOVA with a post-test for subsequent individual group comparisons. A P-value of $<0.05$ was defined as significant.

\section{Results}

miR-21 is overexpressed in human HCC tissues/cell lines and is associated with tumor metastasis. To investigate the biological roles of miR-21 in human HCC development, we first examined the expression of miR-21 in 16 paired human HCC tissues and adjacent non-tumor hepatic tissues by miRNA-based qRT-PCR. Consistent with previous reports (16), miR-21 was significantly upregulated in HCC tissues, as compared with that detected in matched non-tumor tissues (Fig. 1A), suggesting that upregulation of miR-21 might be involved in human HCC development.

These $16 \mathrm{HCC}$ tissues were further divided into an invasive/ metastatic group $(\mathrm{n}=8)$ and a non-invasive/non-metastatic group $(\mathrm{n}=8)$, according to intrahepatic metastases, vascular invasion, or capsular invasion. When comparing miR-21 expression between these two groups, we observed significantly higher levels in the invasive/metastatic group (Fig. 1B). Specifically, upregulation of miR-21 was observed in all nine patients with stage III-IV disease, as compared with that in patients with stage I-II disease (Fig. 1C). We next determined whether miR-21 expression was similarly correlated with the invasiveness of HCC cells lines. We compared miR-21 expression in four selected cell lines (MHCC97H, Bel7402, HepG2 and 

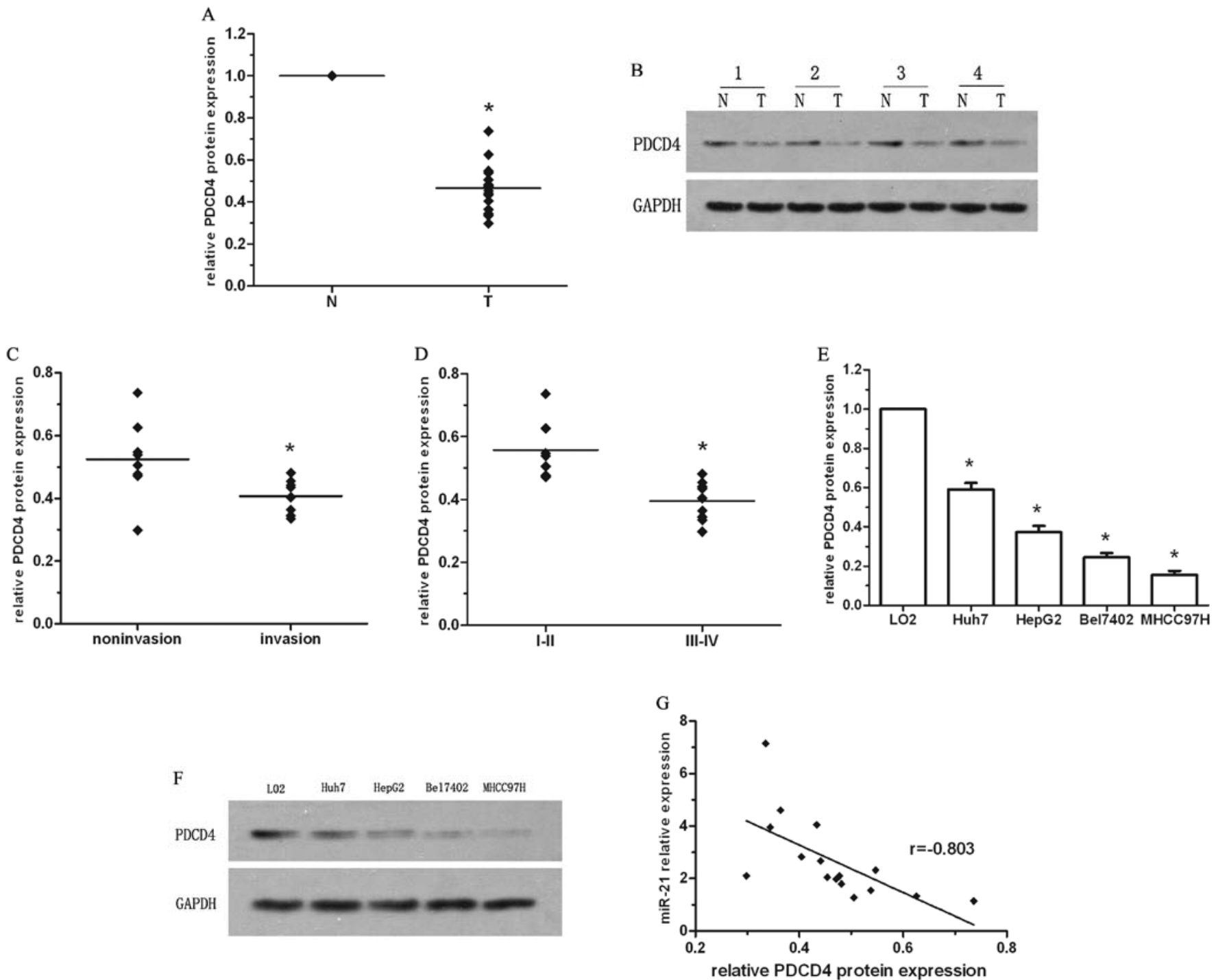

Figure 2. Expression of PDCD4 protein was downregulated in HCC tissues/cell lines with increasing invasive capacity and was inversely correlated to miR-21 levels. (A) Relative PDCD4 protein levels in 16 paired HCC tissues as detected by western blotting. The expression levels of PDCD4 protein relative to GAPDH protein were normalized to the expression of the adjacent non-tumor tissues of each matched pair. N, non-tumor tissue; T, tumor tissue. (B) Representative immunoblot of PDCD4 protein in tumor tissues and adjacent non-tumor tissues. (C). PDCD4 protein was significantly downregulated in invasive/metastatic HCC tissues. (D) PDCD4 protein was significantly downregulated in patients with stage III-IV disease. (E) PDCD4 protein expression was significantly decreased in HCC cell lines, as compared to normal hepatic cells (L02), and was negatively correlated with cell invasiveness. (F) Representative immunoblot of PDCD4 protein in HCC cell lines and normal hepatic cells from three independent experiments. (G) miR-21 expression was inversely correlated with PDCD4 protein in HCC tissues (Pearson's correlation $-0.803, \mathrm{P}=0.007)$. ${ }^{*} \mathrm{P}<0.05$.

Huh7) with increasing invasive capacity (22). Indeed, miR-21 was highly upregulated in HCC cell lines, as compared with human normal hepatic L02 cells, and the miR-21 levels were gradually upregulated in the HCC cell lines with increasing invasive capacity (MHCC97H >Bel7402>HepG2>Huh7) (Fig. 1D). Taken together, these data indicated that miR-21 overexpression was significantly associated with the metastasis and invasiveness of HCC.

$m i R-21$ and PDCD4 protein are inversely expressed in human HCC tissues/cell lines. PDCD4 is a previously identified direct target gene of miR-21 and the miR-21-PDCD4 axis has been implicated in the proliferation and apoptosis of pancreatic cancer and glioblastoma cells $(19,24)$. To analyze the clinical significance of miR-21-regulated PDCD4 in HCC, we examined its protein expression in the 16 pairs of matched
HCC specimens by western blotting. In comparison with the non-tumor counterparts, lower levels of PDCD4 were detected in tumor tissues (Fig. 2A), and representative examples are shown in Fig. 2B. Furthermore, PDCD4 protein was downregulated in HCC samples with invasion/metastasis (Fig. 2C) and in patients with stage III-IV disease (Fig. 2D). Among the total 16 pairs of HCC tissues, we observed a significant inverse correlation between PDCD4 protein expression levels and miR-21 levels ( $\mathrm{P}=0.007$ ) (Fig. 2G). In addition, we also analyzed the expression of PDCD4 protein in normal hepatic cells and the above four HCC cell lines. In agreement with the results from tumor tissues, we observed that the levels of PDCD4 in the $4 \mathrm{HCC}$ cell lines were much lower than that in human normal hepatic cells (Fig. 2E and F), and that PDCD4 expression was negatively correlated with the cell invasive capacity and miR-21 ( $\mathrm{P}=0.002)$ (data not shown). These 


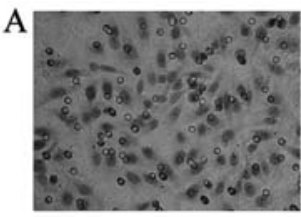

MOCK

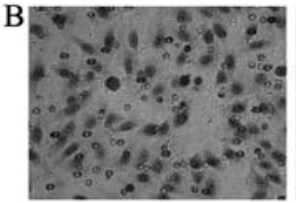

MOCK

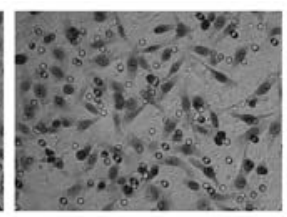

NC

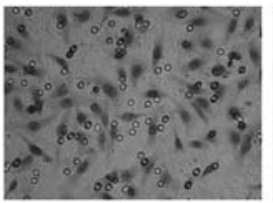

NC

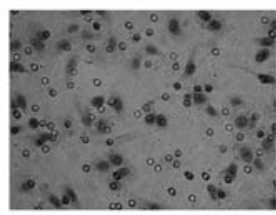

miR-21 inhibitor

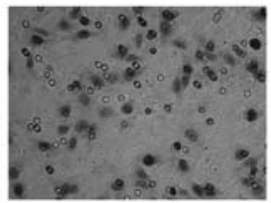

miR-21 inhibitor

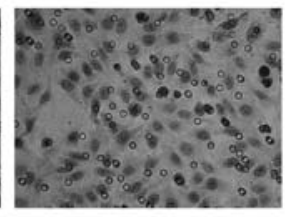

PDCD4 siRNA

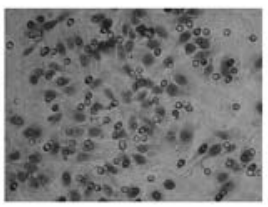

PDCD4 siRNA

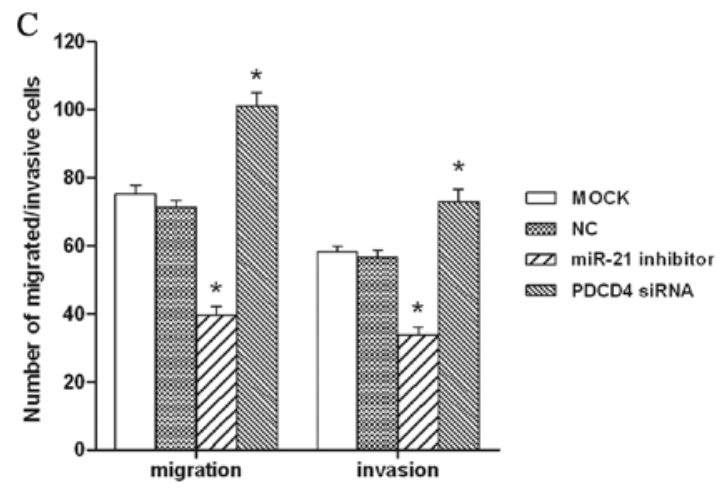

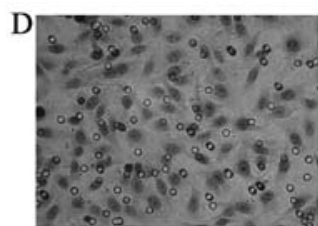

Inhibitor NC + siRNA NC

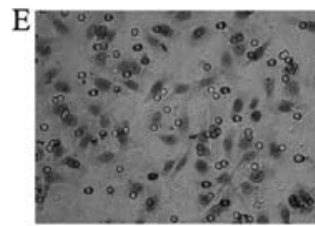

Inhibitor NC+ siRNA NC

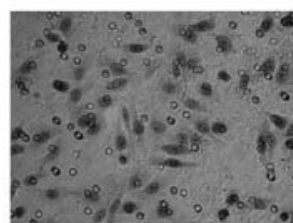

miR-21 inhibitor + siRNA NC

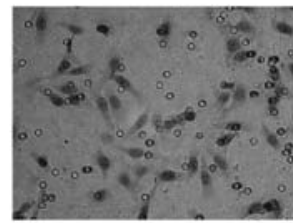

miR-21 inhibitor + siRNA NC

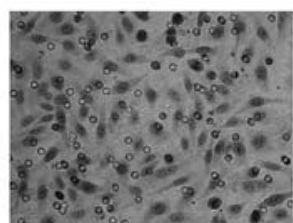

miR-21 inhibitor + PDCD4 siRNA

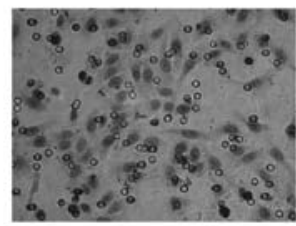

miR-21 inhibitor + PDCD4 siRNA

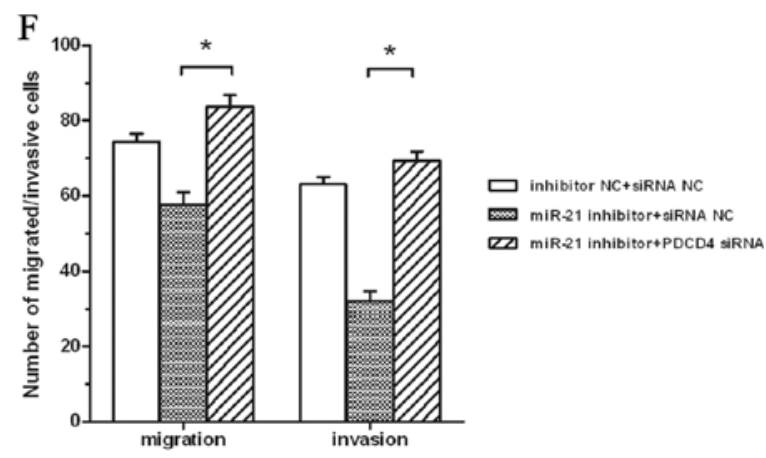

Figure 3. miR-21 promoted migration and invasion in HepG2 cells through PDCD4. (A-C) HepG2 cells were transfected with MOCK, NC, miR-21 inhibitor, and PDCD4 siRNA for $48 \mathrm{~h}$. Transwell cell migration/invasion assays were performed with the cells, as detailed in Materials and methods. Representative images of (A) cell migration and (B) cell invasion from three independent experiments are shown and (C) relative migrated/invaded cells from the above assays were graphed (mean \pm SEM). Compared with NC, miR-21 inhibitor transfection repressed migration/invasion of HepG2 cells, whereas PDCD4 siRNA had the inverse effect. (D-F) HepG2 cells were co-transfected with combinations of the miR-21 inhibitor, inhibitor NC, PDCD4 siRNA and siRNA NC. Cell migration and invasion were detected at $72 \mathrm{~h}$ post-transfection, as described in the Materials and methods. Representative images of (D) migration and (E) invasion from three independent experiments are shown and relative migrated/invaded cells from (F) the above assays were graphed (mean \pm SEM). ${ }^{*} \mathrm{P}<0.05$. 

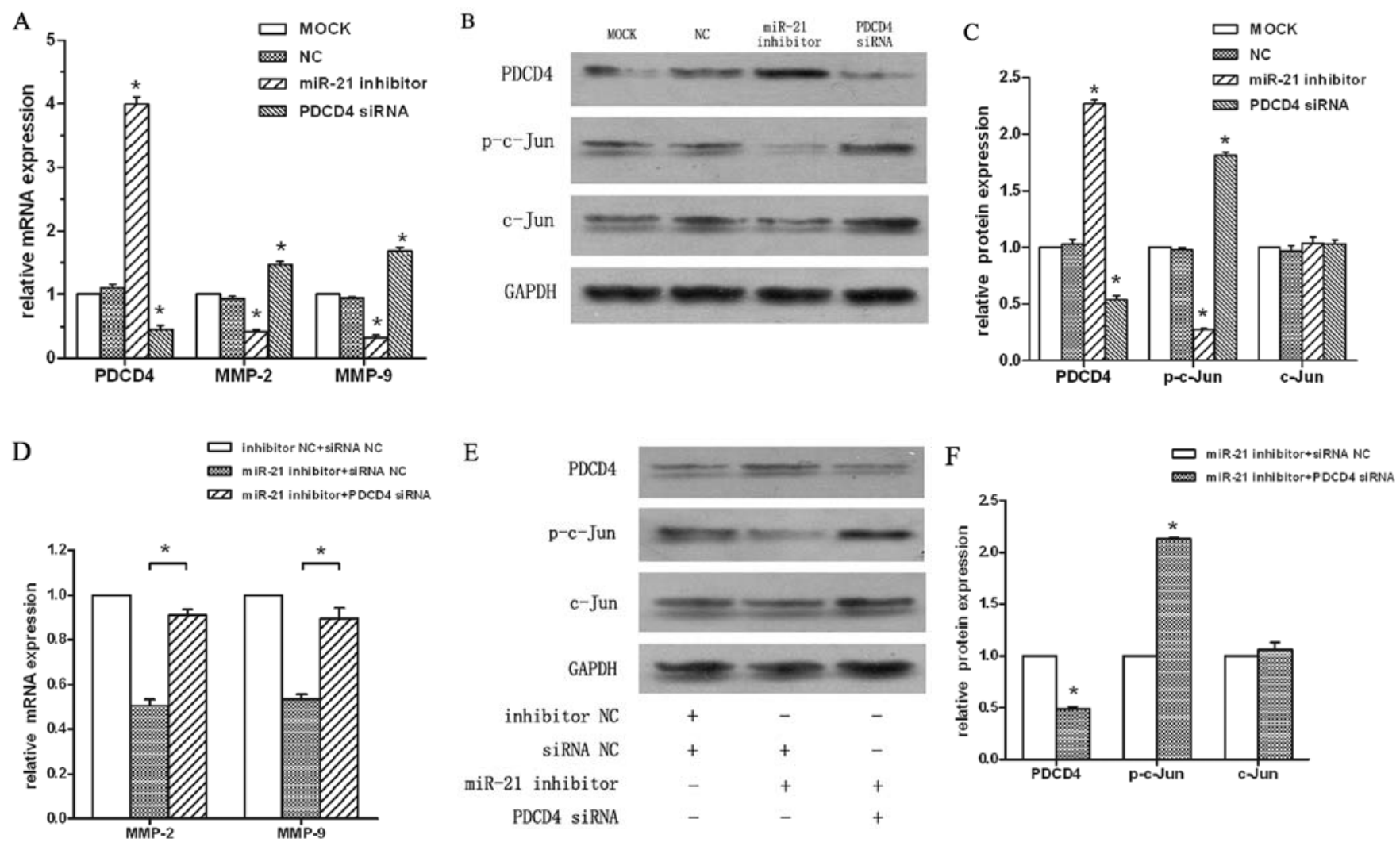

Figure 4. miR-21 suppressed the expression of PDCD4, c-Jun activation, and the MMP downstream signaling molecules. (A-C) HepG2 cells were respectively transfected with MOCK, NC, miR-21 inhibitor or PDCD4 siRNA. (A) After $48 \mathrm{~h}$, total-RNA was isolated and analyzed for PDCD4, MMP-2 and MMP-9 expressions by qRT-PCR. PDCD4, phospho-c-Jun and c-Jun were detected from (B) the same cells by western blotting, and data from (C) three separate experiments were plotted. (D-F) HepG2 cells were co-transfected with combinations of the miR-21 inhibitor, inhibitor NC, PDCD4 siRNA and siRNA NC. (D) The mRNA expression of MMP-2 and MMP-9 were examined by qRT-PCR normalized to that of GAPDH mRNA in the same sample. (E) PDCD4, phospho-c-Jun, and c-Jun levels were evaluated by western blotting, and data from (F) three independent experiments were plotted as mean \pm SEM. ${ }^{*} \mathrm{P}<0.05$.
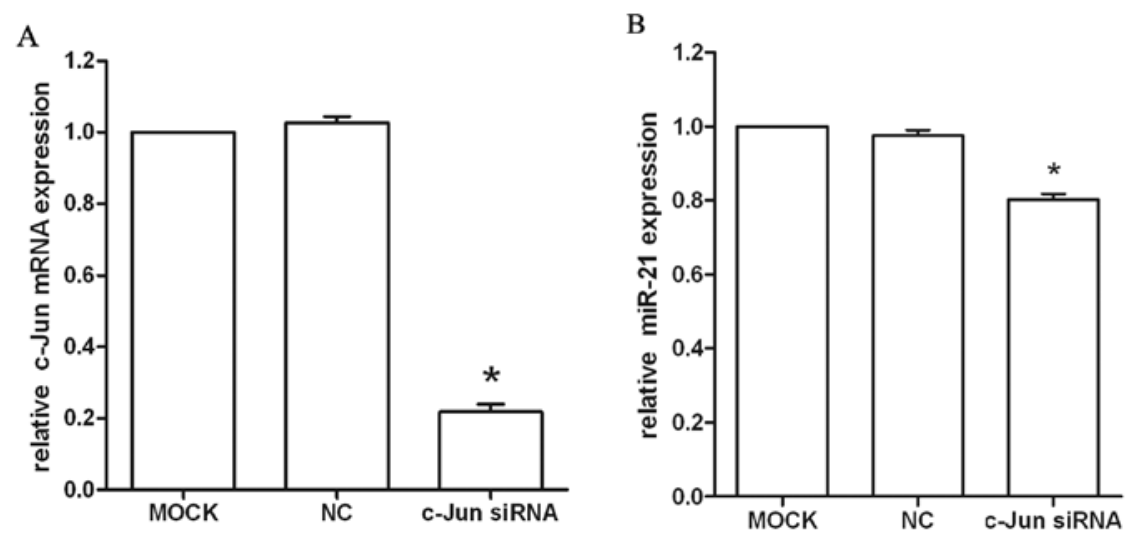

Figure 5. Knockdown of c-Jun inhibited miR-21 expression in HCC cells. HepG2 cells were transfected with MOCK, NC, or c-Jun siRNA for 48 h. (A) c-Jun mRNA and (B) miR-21 expression were detected by qRT-PCR and normalized to GAPDH or U6 snRNA expression, respectively. Data are presented as means of three separate experiments. ${ }^{*} \mathrm{P}<0.05$.

in vitro and in vivo data suggest that downregulation of the miR-21 target gene, PDCD4, might also contribute to tumor metastasis/invasion in HCC.

miR-21 regulates migration and invasion of HepG2 cells through targeting PDCD4. Previous studies have found that a miR-21 inhibitor could suppress cell invasion in some malignant cells by promoting translation of specific target genes $(8,13,14,16)$. Therefore, we aimed to determine whether
miR-21 could have the same impact on HCC cells. HepG2 cells were transfected with blank control culture medium (MOCK), inhibitor NC, or miR-21 inhibitor, in order to evaluate cell migration/invasion activity. The miR-21 inhibitor significantly decreased migration/invasion of HepG2 cells, as compared with NC (Fig. 3A-C), indicating that miR-21 positively regulates migration and invasion of cultured HCC cells. Interestingly, knockdown of PDCD4 in HepG2 cells significantly promoted their migration and invasion (Fig. 3A-C). 
To further evaluate the contribution of PDCD4 to miR21-dependent HCC cell migration and invasion, the HepG2 cells were co-transfected with miR-21 inhibitors and PDCD4 siRNAs, and examined for their migration and invasion capacities. The effect of miR-21 inhibition on decreasing both cell migration and invasion was reversed by the presence of PDCD4 siRNA in HepG2 cells, indicating that PDCD4 was required for the miR-21-dependent cell migration and invasion effect in HCC (Fig. 3D-F).

The miR-21 modulates AP-1 signaling in HCC cells by targeting PDCD4. Gene expression is modulated by multiple mechanisms at different levels. To examine whether downregulation of miR-21 affected the expression of PDCD4 in HCC, HepG2 cells were transiently transfected with the miR-21 inhibitor, NC, or MOCK. The decrease in endogenous miR-21 levels by miR-21 inhibitor significantly increased both PDCD4 mRNA and protein expression levels (Fig. 4A-C). c-Jun, one of the components of transcription factor AP-1, is an important downstream target of PDCD4 and involved in cell apoptosis, proliferation, invasion, and metastasis $(29,30)$. We, thus, compared the levels of total and phosphorylated c-Jun in HepG2 cells following miR-21 inhibition. The expression of phosphorylated c-Jun was decreased by the miR-21 inhibitor. Interestingly, however, total c-Jun protein remained the same (Fig. 4B and C).

Matrix metalloproteinases (MMPs), among which MMP-2 and MMP-9 are the major factors, play an important role in tumor metastasis. PDCD4 suppresses the transcription of several MMPs, including MMP-2 and MMP-9, through downregulation of AP-1 activation (28). Indeed, mRNA expression of both MMP-2 and MMP-9 was significantly decreased after transfection with miR-21 inhibitor in HepG2 cells, but was further enhanced by PDCD4 knockdown (Fig. 4A), suggesting that miR-21 may exert its biological functions through PDCD4dependent AP-1 signaling. Therefore, we next investigated the ability of PDCD4 to modulate miR-21-mediated inhibition of MMP-2 and MMP-9 mRNA expression. As expected, miR-21 inhibition-induced downregulation of MMP-2, MMP-9 and phosphorylated c-Jun was reversed by PDCD4 knockdown in HepG2 cells (Fig. 4D-F). Together, these data suggest an important role of PDCD4 as a mediator of the biological effects of miR-21.

AP-1 activates miR-21 transcription. The transcriptional factor AP-1 activates gene transcription by binding to its promoter region. Bioinformatic analysis suggested that AP-1 binding sites are enriched in the putative promoter region of miR-21 (37). To evaluate the regulation of miR-21 expression by c-Jun, HepG2 cells were transfected with c-Jun siRNA and examined for miR-21 expression. miR-21 levels were significantly downregulated in cells transfected with c-Jun siRNA, as compared to control cells (Fig. 5), suggesting that AP-1 activates miR-21 transcription.

\section{Discussion}

As an oncomir, miR-21 is upregulated in a variety of human malignancies, such as cholangiocarcinoma, breast cancer, lung cancer, colorectal cancer, and prostatic carcinoma
(8-12). Consistent with these studies, we demonstrated that miR-21 expression was significantly higher in HCC tissues and cell lines than that in matched non-tumor tissues and normal human hepatic cells, suggesting that high miR-21 expression was tightly associated with tumor development. Inhibition of miR-21 expression could restrain tumor growth in breast cancer cells (9), while overexpression of miR-21 could increase cell invasion in colon carcinoma (12). In this study, we observed that miR-21 expression was dramatically increased in invasive/metastatic HCC tumor tissues and tumor tissues from stage II-IV patients, as compared with that in non-invasive/non-metastatic HCC tumor tissues and tumor tissues from stage I-II patients. We also observed that miR-21 expression was gradually increased in HCC cell lines, along with increased invasive capacity, and inhibition of miR-21 expression could restrain migration and invasion of HepG2 cells. These findings suggest that overexpression of miR-21 was associated with advanced TNM stage and metastasis in HCC.

Tumor suppressor PDCD4 is one of the miR-21 target genes (8). PDCD4 expression is usually reduced or is absent from many human malignancies, including renal-, lung- and gliaderived tumors $(23,25)$. Consistent with these findings, we also observed that the expression of PDCD4 protein in HCC tissues and cell lines was significantly lower than that in matched nontumor tissues and human normal hepatic cells. In addition, a highly significant negative correlation between miR-21 expression and PDCD4 protein levels in HCC tumor tissues and cell lines existed. Meanwhile, as opposed to miR-21 expression, PDCD4 protein expression was significantly downregulated in metastatic HCC tumor tissues and tumor tissues from stage III-IV patients, and was gradually reduced in HCC cell lines along with increasing invasive capacity. Inhibition of PDCD4 expression could promote migration and invasion of HepG2 cells. Taken together, these data suggest that PDCD4 is a critical mediator through which miR-21 exerts its biological functions in HCC.

Interestingly, a recent study has demonstrated that PDCD4 modulates cancer cell proliferation and invasion by regulating different downstream genes in a cell type/ tissue type-dependent manner. PDCD4 inhibited u-PAR expression and cell invasion and metastasis via SP1/SP3 in colorectal carcinoma cells (17), but in breast cancer cells, PDCD4 overexpression did not affect u-PAR transcription and inhibited cell invasion by increasing TIMP-2 secretion (26). PDCD4 inhibits c-Jun activation and AP-1-dependent transcription via the downstream MAP4K1/JNK/AP-1 signaling pathway in colon carcinoma cells $(27,28)$. AP-1 is a transcription factor complex comprised of Jun-Jun homodimers or Jun-Fos heterodimers (29). The Jun protein family includes c-Jun, JunB and JunD. The Fos protein family contains c-Fos, Fra-1, Fra-2 and FosB. c-Jun is a key molecule in the AP-1 complex. AP-1 regulates multiple biological events required for cell invasion, including expression of the MMPs (30-32) and cell motility $(33,34)$. Overexpression or hyperactivation of AP-1 components enhances invasion $(35,36)$. In the present study, we reported that miR-21 regulated PDCD4 expression at the post-transcriptional and translational levels in HepG2 cells, increased phosphorylation of c-Jun protein without affecting its synthesis, activated AP-1-dependent transcription of 
MMP-2 and MMP-9 and promoted migration and invasion of HCC cell lines. Interestingly, we showed that HepG2 cell migration and invasiveness weakened by the miR-21 inhibitor was reversed by knockdown of PDCD4, and that downregulation of the AP-1 component (phospho-c-Jun) and its downstream signaling molecules (MMP-2 and MMP-9) was also rescued by knockdown of PDCD4. These findings supported the hypothesis of PDCD4 playing a critical role in mediating miR-21 dependent biological functions in HCC.

Several conserved enhanced elements have been identified in the consensus sequence of the putative promoter region of miR-21 (miPPR-21) based on TRANSFAC matrices, including binding sites for AP-1, Ets/PU.1, C/EBP $\alpha$, NFI, SRF, p53 and STAT3 (37). Fujita et al (37) reported that AP-1 was capable of activating miR-21 transcription through the conserved AP-1 and PU.1 binding sites in the miPPR-21 after PMA stimulation. In support of the notion that AP-1 could directly activate miR-21 transcription, in this study we showed that miR-21 was downregulated in HepG2 cells transfected with siRNA to c-Jun. Therefore, a positive feedback loop mediated by miR-21-PDCD4-AP-1 could control the AP-1-dependent oncogenes activity, which might be partially involved in invasion and metastasis in HCC. Certainly, additional mechanisms and regulators of the loop are also likely to contribute to miR-21-induced HCC cell invasion, which needs to be further elucidated.

In conclusion, we demonstrated that miR-21 was overexpressed in HCC tissues and cell lines and was a potent stimulator of the migrative/invasive capacities of HCC cells. We further showed that a miR-21 inhibitor restrained HepG2 cell migration and invasion through direct/indirect regulation of PDCD4 and downstream signaling pathway molecules (p-cJun/AP-1, MMP-2 and MMP-9), and that the transcription factor AP-1 directly activates miR-21 transcription. We, thus, hypothesize that a positive feedback loop of miR-21-PDCD4AP-1 maintains the miR-21-mediated biological effects of the HCC malignant phenotype. Our data suggest that molecular introduction strategies interfering with the miR-21-PDCD4AP-1 feedback loop might provide strong rationale for preventing invasion/metastasis in $\mathrm{HCC}$ in the future.

\section{Acknowledgements}

We are grateful to Dr Yanning Huang for editorial assistance. We thank Dr Jinfeng Xue and Dr Yu Peng for technical assistance.

\section{References}

1. Hanazaki K, Kajikawa S, Shimozawa N, et al: Survival and recurrence after hepatic resection of 386 consecutive patients with hepatocellular carcinoma. J Am Coll Surg 191: 381-388, 2000.

2. Sanefuji K, Taketomi A, Iguchi T, Sugimachi K, Ikegami T and Yamashita Y: Significance of DNA polymerase delta catalytic subunit p125 induced by mutant p53 in the invasive potential of human hepatocellular carcinoma. Oncology 79: 229-237, 2010.

3. Endo K, Ueda T, Ohta T and Terada T: Protein expression of MDM2 and its clinicopathological relationships in human hepatocellular carcinoma. Liver 20: 209-215, 2000.

4. Onishi T, Nouso K, Higashi T, Toshikuni N, Nakatsukasa H and Kobayashi Y: Cellular distribution of telomerase reverse transcriptase in human hepatocellular carcinoma. J Gastroenterol Hepatol 18: 1168-1174, 2003.
5. Yuan RH, Jeng YM, Hu RH, Lai PL, Lee PH and Cheng CC: Role of p53 and $\beta$-catenin mutations in conjunction with CK19 expression on early tumor recurrence and prognosis of hepatocellular carcinoma. J Gastrointest Surg 15: 321-329, 2011.

6. Shim JH, Park JW, Kim JH, An M and Kong SY: Association between increment of serum VEGF level and prognosis after transcatheter arterial chemoembolization in hepatocellular carcinoma patients. Cancer Sci 99: 2037-2044, 2008.

7. Valencia-Sanchez MA, Liu J, Hannon GJ and Parker R: Control of translation and mRNA degradation by miRNAs and siRNAs. Genes Dev 20: 515-524, 2006.

8. Selaru FM, Olaru AV, Kan T, David S and Cheng Y: MicroRNA-21 is overexpressed in human cholangiocarcinoma and regulates programmed cell death 4 and tissue inhibitor of metalloproteinase 3. Hepatology 49: 1595-1601, 2009.

9. Rask L, Balslev E, Jørgensen S, Eriksen J, Flyger H and Møller S: High expression of miR-21 in tumor stroma correlates with increased cancer cell proliferation in human breast cancer. APMIS 119: 663-673, 2011.

10. Lee JH, Voortman J, Dingemans AM, Voeller DM and Pham T: MicroRNA expression and clinical outcome of small cell lung cancer. PLoS One 6: 1300-1308, 2011.

11. Leite KR, Tomiyama A, Reis ST, Sousa-Canavez JM and Sañudo A: MicroRNA expression profiles in the progression of prostate cancer-from high-grade prostate intraepithelial neoplasia to metastasis. Urol Oncol 29: 101-107, 2011.

12. Cottonham CL, Kaneko S and Xu L: miR-21 and miR-31 converge on TIAM1 to regulate migration and invasion of colon carcinoma cells. J Biol Chem 285: 35293-35302, 2010.

13. Zhu S, Si ML, Wu H and Mo YY: MicroRNA-21 targets the tumor suppressor gene tropomyosin 1 (TPM1). J Biol Chem 282: 14328-14336, 2007.

14. Zhu S, Wu H, Wu F, Nie D, Sheng S and Mo YY: MicroRNA-21 targets tumor suppressor genes in invasion and metastasis. Cell Res 18: 350-359, 2008.

15. Sayed D, Rane S, Lypowy J, He M, Chen IY, Vashistha H, Yan L, Malhotra A, Vatner D and Abdellatif M: MicroRNA-21 targets Sprouty2 and promotes cellular outgrowths. Mol Biol Cell 19: 3272-3282, 2008.

16. Meng F, Henson R, Janek H, Ghoshal K, Jacob S and Patel T: MicroRNA-21 regulates expression of the PTEN tumor suppressor gene in human hepatocellular cancer. Gastroenterology 133: 647-658, 2007.

17. Leupold JH, Yang HS, Colburn NH, Asangani I, Post S and Allgayer H: Tumor suppressor Pdcd4 inhibits invasion/intravasation and regulates urokinase receptor (u-PAR) gene expression via Sp-transcription factors. Oncogene 26: 4550-4562, 2007.

18. Zhang H, Ozaki I, Mizuta $\mathrm{T}$ and Hamajima $\mathrm{H}$ : Involvement of programmed cell death 4 in transforming growth factor-b1-induced apoptosis in human hepatocellular carcinoma. Oncogene 25: 6101-6112, 2006.

19. Bhatti I, Lee A, James V, Hall RI, Lund JN, Tufarelli C, et al: Knockdown of miR-21 inhibits proliferation and increases cell death by targeting PDCD4 in pancreatic ductal adenocarcinoma. J Gastrointest Surg 15: 199-208, 2011.

20. International Union Against Cancer (UICC). TNM Classification of Malignant Tumors. Sobin LH and Witterkind C (eds). 6th edition, Wiley-Liss, New York, pp81-83, 2002.

21. Pfaffl MW: A new mathematical model for relative quantification in real-time RT-PCR. Nucleic Acids Res 29: 2002-2007, 2001.

22. Lee TK, Man K, Ho JW, Wang XH, Poon TP, Xu Y, et al: FTY720: a promising agent for treatment of metastatic hepatocellular carcinoma. Clin Cancer Res 11: 8458-8466, 2005.

23. Carinci F, Lo ML, Piattelli A, Rubini C, Chiesa F, Ionna F, Palmieri A, Maiorano E, Pastore A, Laino G, et al: Potential markers of tongue tumor progression selected by cDNA microarray. Int J Immunopathol Pharmacol 18: 513-524, 2005.

24. Chen Y, Liu W, Chao T, Zhang Y, Yan X, et al: MicroRNA-21 downregulates the expression of tumor suppressor PDCD4 in human glioblastoma cell T98G. Cancer Lett 18: 197-205, 2008.

25. Jansen AP, Camalier CE, Stark C and Colburn NH: Characterization of programmed cell death 4 in multiple human cancers reveals a novel enhancer of drug sensitivity. Mol Cancer Ther 3: 103-110, 2004.

26. Nieves-Alicea R, Colburn NH, Simeone AM and Tari AM: Programmed cell death 4 inhibits breast cancer cell invasion by increasing tissue inhibitor of metalloproteinases-2 expression. Breast Cancer Res Treat 114: 203-209, 2009. 
27. Bitomsky N, Bohm M and Klempnauer KH: Transformation suppressor protein Pdcd4 interferes with JNK-mediated phosphorylation of c-Jun and recruitment of the coactivator p300 by c-Jun. Oncogene 23: 7484-7493, 2004.

28. Yang HS, Matthews CP, Clair T, Wang Q, Baker AR, Li CC Tan TH and Colburn NH: Tumorigenesis suppressor Pdcd4 downregulates mitogen-activated protein kinase kinase kinase kinase 1 expression to suppress colon carcinoma cell invasion. Mol Cell Biol 26: 1297-1306, 2006.

29. Shaulian E and Karin M: AP-1 in cell proliferation and survival. Oncogene 20: 2390-2400, 2001.

30. Benbow U and Brinckerhoff CE: The AP-1 site and MMP gene regulation: what is all the fuss about? Matrix Biol 15: 519-526, 1997.

31. Crawford HC and Matrisian LM: Mechanisms controlling the transcription of matrix metalloproteinase genes in normal and neoplastic cells. Enzyme Protein 49: 20-37, 1996.

32. Curran S and Murray GI: Matrix metalloproteinases: molecular aspects of their roles in tumour invasion and metastasis. Eur J Cancer 36: 1621-1630, 2000.
33. Pilcher BK, Dumin JA, Sudbeck BD, Krane SM, Welgus HG and Parks WC: The activity of collagenase-1 is required for keratinocyte migration on a type I collagen matrix. J Cell Biol 137: 1445-1457, 1997.

34. Shin EY, Kim SY and Kim EG: c-Jun N-terminal kinase is involved in motility of endothelial cell. Exp Mol Med 33: 276-283, 2001.

35. Hennigan RF, Hawker KL and Ozanne BW: Fos-transformation activates genes associated with invasion. Oncogene 9: 3591-3600, 1994.

36. Marconcini L, Marchio S, Morbidelli L, Cartocci E, Albini A, Ziche M, Bussolino F and Oliviero S: c-Fos-induced growth factor/vascular endothelial growth factor $\mathrm{D}$ induces angiogenesis in vivo and in vitro. Proc Natl Acad Sci USA 96: 9671-9676, 1999.

37. Fujita S, Ito $\mathrm{T}$, Mizutani $\mathrm{T}$, Minoguchi S, Yamamichi $\mathrm{N}$ and Sakurai K: miR-21 gene expression triggered by AP-1 is sustained through a double-negative feedback mechanism. J Mol Biol 378: 492-504, 2008 